Tema: Diagramas de fases e transformações de fases

\title{
APLICAÇÃO DA TERMODINÂMICA COMPUTACIONAL NO PROCESSAMENTO E TRATAMENTOS TÉRMICOS DE AÇOS ELÉTRICOS COM 2-3\%SI*
}

\author{
Leandro Nakamura Alves Vieira ${ }^{1}$ \\ André Luiz Vasconcellos da Costa e Silva²
}

\section{Resumo}

O acirramento da competição no mercado de aço tem exigido esforços de inovação e redução de custos. Por sua vez, as técnicas agrupadas como ICME - Integrated Computational Materials Engineering vêm acelerando o desenvolvimento e a otimização de materiais e processos de fabricação. O emprego destas técnicas tem sido uma ferramenta extra para assegurar a competitividade na indústria. Neste trabalho o uso da termodinâmica computacional no desenvolvimento e processamento de aços elétricos é revisado através da discussão de exemplos. Cálculos de equilíbrios mais relevantes para o processo de fabricação destes aços (tanto de grão não orientado-GNO como orientado-GO) permitem obter informações sobre as transformações de fases, solubilização de precipitados (sulfetos e nitretos) essenciais para a obtenção de texturas cristalográficas ideais. Os cálculos são discutidos e comparados com resultados reais. Discutem-se ainda, cálculos no sistema açogás, e os efeitos da composição da atmosfera na formação de óxidos na superfície do aço. Tais cálculos são importantes para descrever previamente as transformações que ocorrem durante as várias etapas de fabricação, assim como os efeitos dos parâmetros de processo sob os aços elétricos. A aplicação da técnica permite otimizar o processamento e o desenvolvimento dos aços elétricos em diversos aspectos. As vantagens e limitações do método, assim como o potencial de outras técnicas de ICME, na área, são discutidas.

Palavras-chave: Aços elétricos; Termodinâmica computacional; Transformação de fases, Oxidação.

\section{APPLICATION OF COMPUTATIONAL THERMODYNAMICS IN PROCESSING AND HEAT TREATMENTS OF 2-3WT.\% ELECTRICAL STEELS}

\begin{abstract}
The competition in the steel market has demanding innovation and cost reduction efforts. On the other hand, approaches such as ICME - Integrated Computational Materials Engineering has receiving international attention due to its great potential to shorten product and process development time, while lowering cost and improving outcome. In this work, the use of computational thermodynamics in the development and processing of electrical steels is reviewed by examples discussions. Equilibrium calculations for relevant manufacturing process of electrical steels (grain non-oriented GNO and grain oriented GO) provided information about the phase transformations and precipitate solubilization (nitrides and sulfides) for several chemical compositions. Such parameters are essential to drive the pathway to get ideal crystallographic textures. The calculations are discussed and compared with experimental results of commercial electrial steels. Furthermore, calculations involving steel-gas system were performed, and the effects of gases compositions in the atmosphere on the formation of oxides in surface layer of the steel were discussed. Such calculations are important for previously describing the transformations that occur during the various stages of manufacturing process, as well as the effects of process parameters on properties of such steel. The application of the technique allows optimizing the process and development of electrical steels in various aspects. The advantages and limitations of the method as well the potential of other techniques ICME are discussed.
\end{abstract}

Keywords: Electrical steels; Computational thermodynamics; Phases transformation; Oxidation.

1 Físico, Mestre, Pesquisador, Centro de Pesquisa, Aperam, Timóteo, MG, Brasil.

2 Engenheiro, PhD, Professor, EEIMVR, UFF, Volta Redonda, RJ, Brasil.

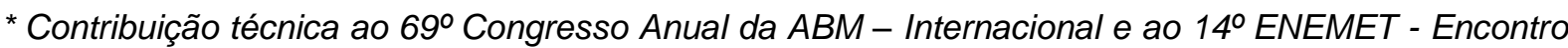
Nacional de Estudantes de Engenharia Metalúrgica, de Materiais e de Minas, 21 a 25 de julho de 2014, São Paulo, SP, Brasil.
} 


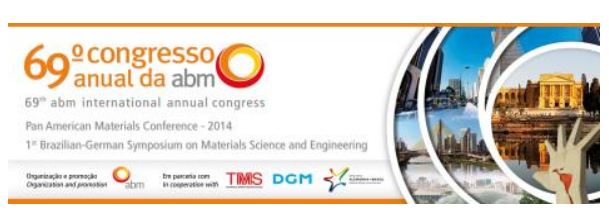

\section{INTRODUÇÃO}

O acirramento da competição global no mercado de aço decorrente do excesso de capacidade de produção e baixo consumo mundial devido à desaceleração da China e demais países emergentes, à crise europeia e às incertezas sobre a política fiscal nos Estados Unidos, têm esmagado a rentabilidade das siderúrgicas [1], fazendo com que a redução de custos e a inovação tornem-se pilares fundamentais para a sustentabilidade econômica e sobrevivência neste mercado. Por sua vez, as técnicas computacionais tal como ICME - Integrated Computational Materials Engineering têm alcançado níveis maduros e recebido atenção internacional especial [2] devido ao grande potencial destas ferramentas tanto para acelerar o desenvolvimento de novos materiais como para a otimização de processos consagrados de fabricação. Desta forma, vários problemas que até então só podiam ser resolvidos empiricamente passaram a ser abordados de forma sistemática, criando uma grande oportunidade para a inovação e redução de custos.

Aços elétricos são materiais magnéticos macios tipicamente utilizados como núcleo de transformadores, motores e geradores elétricos. A principal função desses materiais é conduzir o fluxo magnético quando são submetidos à uma magnetização externa cíclica, com baixa perda para minimizar o consumo de energia elétrica e alta permeabilidade para operar em diferentes condições de magnetização dependendo da aplicação. De modo geral, os aços elétricos podem ser classificados em duas categorias: Aços elétricos de grão não orientado (GNO), processados de modo a desenvolver propriedades magnéticas isotrópicas, por esse motivo são empregados em máquinas elétricas rotativas como motores e geradores. Por outro lado, os aços elétricos de grão orientado (GO) possuem propriedades magnéticas anisotrópicas, sendo assim utilizados em transformadores de energia elétrica. Fatores como: resistividade, tamanho de grão, espessura, pureza da liga e principalmente a textura cristalográfica podem afetar as propriedades magnéticas dos aços elétricos. Além disso, tais fatores estão intimamente relacionados à composição química e ao processamento dos aços elétricos.

Em altas temperaturas, praticadas em processos de aciaria, laminação a quente e alguns tratamentos térmicos posteriores, os materiais alcançam estados termodinâmicos próximos aos de equilíbrio, ou mesmo em outros casos, extrapolações podem ser realizadas [3]. Por esse motivo, o conhecimento da estabilidade das fases presentes e as condições de processamento são essenciais para o desenvolvimento e a otimização dos materiais. Nesta primeira etapa do trabalho, cálculos de diagramas de equilíbrio mais relevantes aplicados às etapas de processo industrial de fabricação dos aços elétricos GNO e GO foram realizados, obtendo informações sobre as transformações de fases primárias (ferrita e austenita) e secundárias (sulfetos, nitretos e etc.), cujo controle morfológico é fundamental para o desenvolvimento de texturas cristalográficas ideais. Foram calculados ainda, diagramas referentes às transformações de fases envolvendo o sistema aço-gás, e os efeitos das atmosferas gasosas ( $\mathrm{H} 2, \mathrm{~N} 2$ e H2O) no processo na formação de óxidos na superfície do aço.

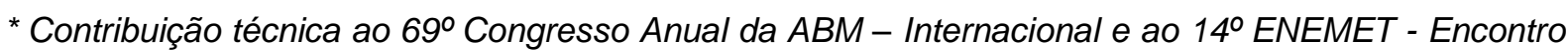
Nacional de Estudantes de Engenharia Metalúrgica, de Materiais e de Minas, 21 a 25 de julho de 2014, São Paulo, SP, Brasil.
} 


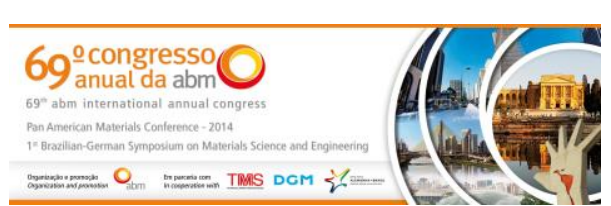

\section{MATERIAIS E MÉTODOS}

O software empregado neste trabalho foi o Thermo-Calc versão 5.0 e os dados termodinâmicos dos vários sistemas estudados foram extraídos das bases de dados TCFE6 - TCS Steels/Fe-Alloys Database v6.2 e SSUB4 - SGTE Substance Database v4.1.

\section{RESULTADOS E DISCUSSÃO}

\subsection{Efeito da Composição Química na Laminação a Quente}

Os processos de fabricação realizados em alto forno e aciaria podem não produzir composições químicas exatamente iguais para um mesmo aço. Essas diferenças são especificadas previamente dentro de faixas limites para cada produto e são geralmente função da natureza da matéria prima utilizada (minérios, aglomerados, combustíveis, redutores, fluxantes e etc) como também das características dos equipamentos utilizados nas etapas de elaboração do aço.

Os aços elétricos GNO mais comercializados tem tipicamente 2,0\% (em massa) de Silício, dependendo da aplicação há outras grades onde este valor pode ser $3,0 \%$ e até $3,3 \%$ [4]. Essa variação juntamente com outros elementos químicos presentes no aço tem forte efeito nas transformações de fase que ocorrem durante a laminação a quente e por consequência influencia a textura cristalográfica nas etapas posteriores de fabricação $[5,6]$.

Os resultados dos cálculos do diagrama de equilíbrio para o aço elétrico GNO que mostram as fronteiras e intersecções das fases: Austenita ( $\gamma$ ou FCC_A1) e Ferrita ( $\alpha$ ou BCC_A2) quando varia-se temperatura e \%Si é mostrado na Figura 1.

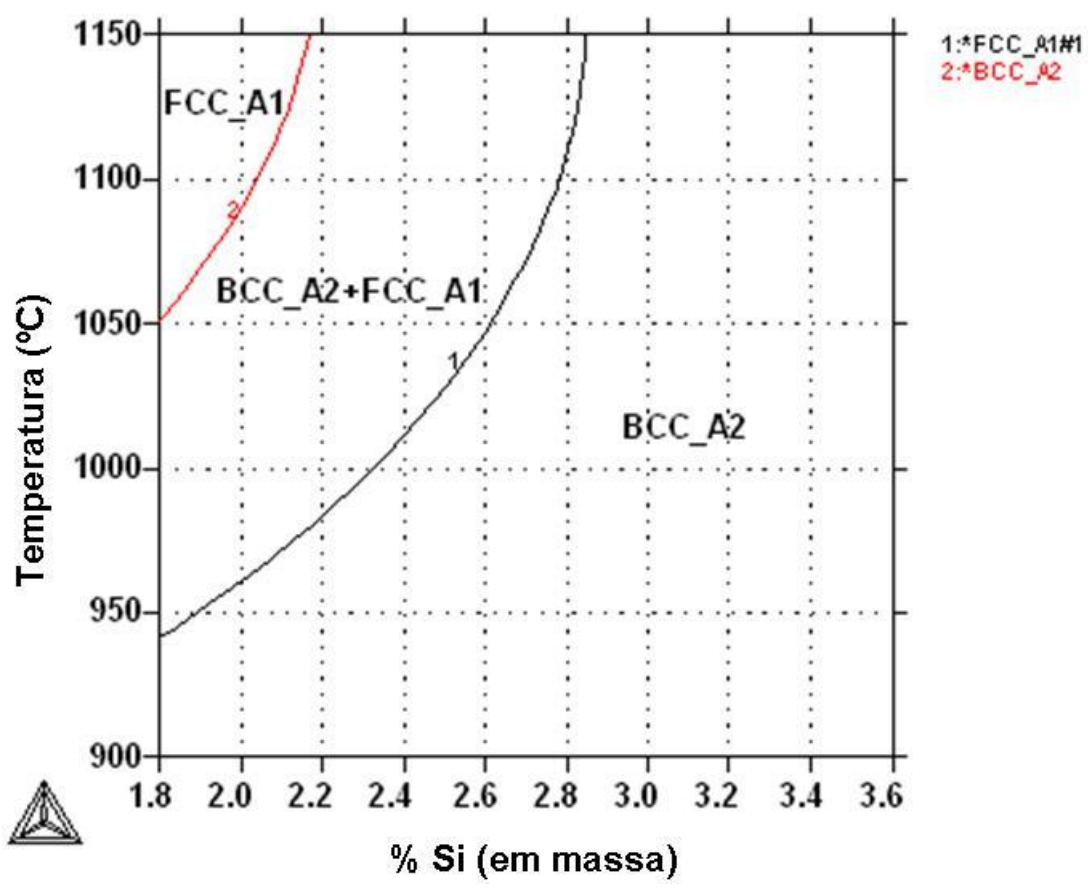

Figura 1. Diagrama de fases do aço elétrico GNO.

Pela Figura 1 pode-se notar que dependendo do teor de Silício os parâmetros de processos operacionais termomecânicos praticados na laminação a quente do aço elétrico GNO precisam ser ajustados em termos de temperatura, carga, tipo de

\footnotetext{
* Contribuição técnica ao 69ำ Congresso Anual da ABM - Internacional e ao 14ํㅡㄹ ENEMET - Encontro Nacional de Estudantes de Engenharia Metalúrgica, de Materiais e de Minas, 21 a 25 de julho de 2014, São Paulo, SP, Brasil.
} 


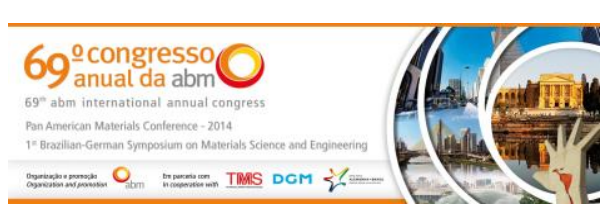

cilindros de laminação entre outros [7], de modo a alcançar melhores propriedades magnéticas no produto final.

Cálculos da fração (em massa) da austenita em função da temperatura para três cenários de composição química diferentes (máximo de $\gamma$, típico e mínimo de $\gamma$ ) foram realizados e os resultados são mostrados na Figura 2.

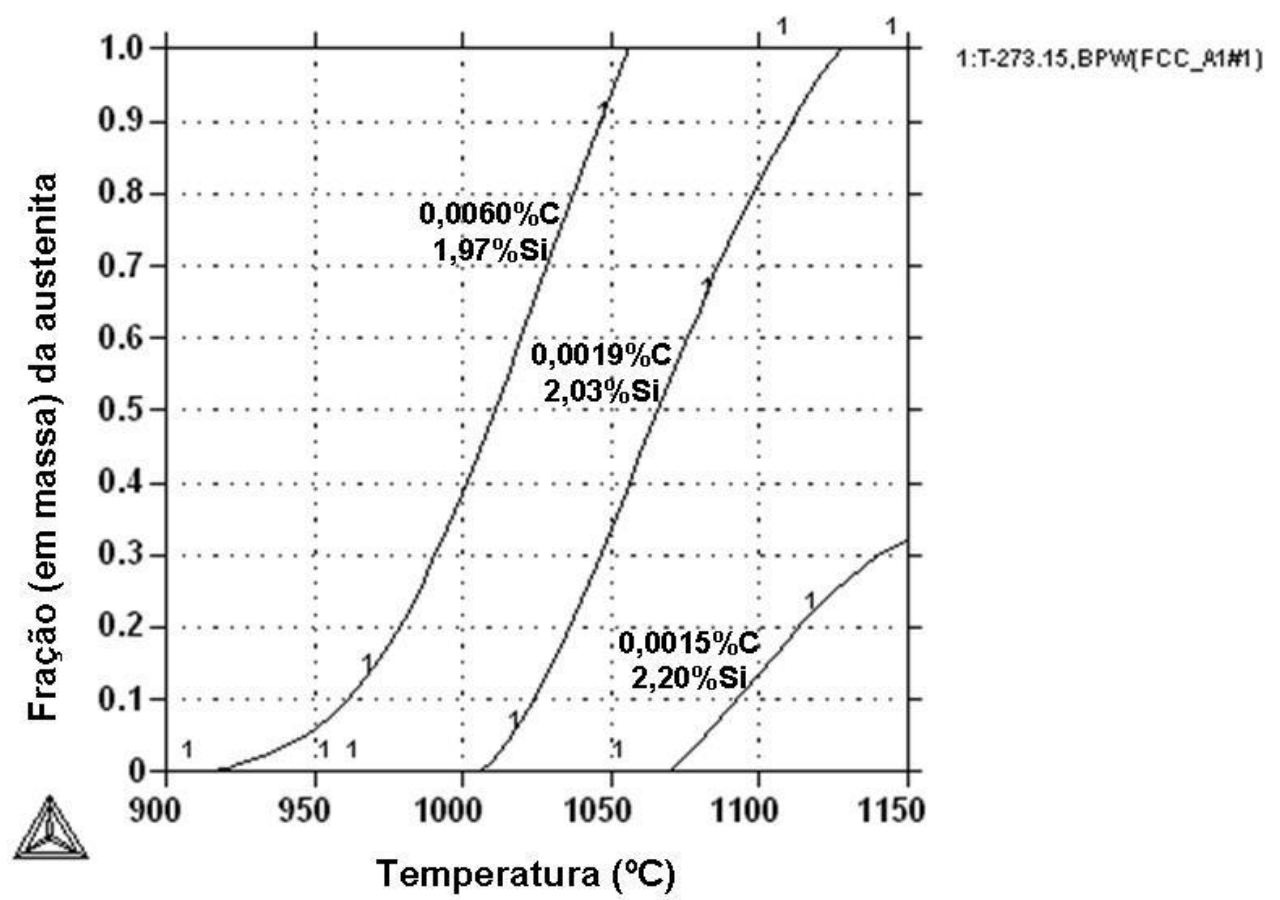

Figura 2. Fração em massa da austenita em função da temperatura para três composições químicas diferentes (máximo de $\gamma$, típico e mínimo de $\gamma$ ) do aço elétrico GNO com 2,0\%Si

Embora os resultados para as três composições químicas calculadas na Fig. 2 estejam dentro da faixa especificada para o aço elétrico GNO com 2,0\% Silício, ajustes na temperatura de laminação a quente precisam ser feitos, principalmente onde as frações de austenita não são desprezíveis, a fim de evitar obtenção de texturas cristalográficas indesejáveis no produto final [8].

A precipitação de inibidores ou partículas de segunda fase são essenciais na tecnologia de produção dos aços elétricos GO, pois tem um profundo efeito sob os processos de recristalização ancorando o crescimento dos grãos para então nas etapas subsequentes, o material desenvolver alta fração volumétrica da textura Goss através do fenômeno de crescimento anormal dos grãos de Goss [9-11]. A magnitude desta interação de ancoramento depende da fração, tamanho e natureza química e cristalográfica dos inibidores [12-13]. Fomentar o desenvolvimento da textura Goss com baixo grau de desalinhamento em relação a direção de laminação é fundamental para alcançar boas propriedades magnéticas neste aço.

A Figura 3 mostra os resultados dos cálculos das temperaturas de solubilização do inibidor sulfeto de manganês (MnS), presentes no o aço elétrico $\mathrm{GO}$, calculadas para várias condições da razão $\mathrm{Mn} / \mathrm{S}$ em função do Manganês do aço. Os resultados sugerem que para um mesmo valor de Manganês, quanto maior a razão Mn/S maior será temperatura para solubilizar estes sulfetos. Por esse motivo, que são empregadas altas temperaturas de reaquecimento de placas neste aço para completa dissolução dos sulfetos provenientes das etapas anteriores.

\footnotetext{
* Contribuição técnica ao $69^{\circ}$ Congresso Anual da ABM - Internacional e ao 14ํㅡㄹ ENEMET - Encontro Nacional de Estudantes de Engenharia Metalúrgica, de Materiais e de Minas, 21 a 25 de julho de 2014, São Paulo, SP, Brasil.
} 


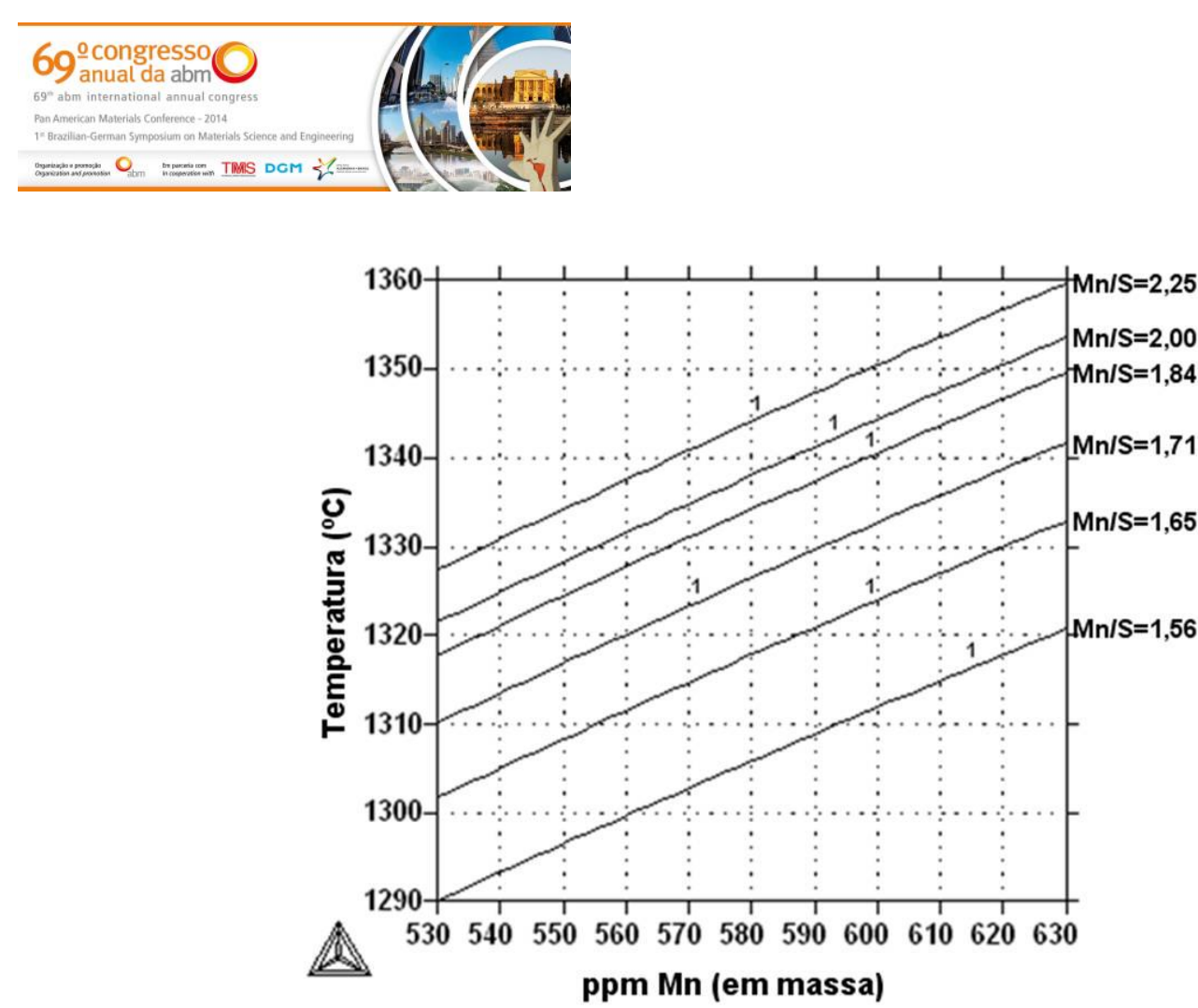

Figura 3. Temperatura de solubilização do inibidor Sulfeto de Manganês calculados para várias razões $\mathrm{Mn} / \mathrm{S}$ em função do ppm de Manganês do aço elétrico GO

Na Figura 4 os resultados dos cálculos da fração (em massa) do MnS são mostrados na condição $\mathrm{Mn} / \mathrm{S}=1,84$ em função do teor Manganês do aço para várias temperaturas diferentes. Note que a medida que a temperatura diminui temos aumento da fração de MnS precipitada. Porém, essa fração dependente do teor de Manganês no aço, e somente em temperaturas abaixo de $1290^{\circ} \mathrm{C}$ é que teremos mais de $10^{-4}$ de fração de inibidores precipitada para toda a faixa de Manganês calculada. Do mesmo modo como pode ser observado na Figura 3, é importante destacar que, mantendo-se a razão $\mathrm{Mn} / \mathrm{S}$ fixa, com o aumento de Manganês do aço, a temperatura de solubilização de MnS também aumenta.

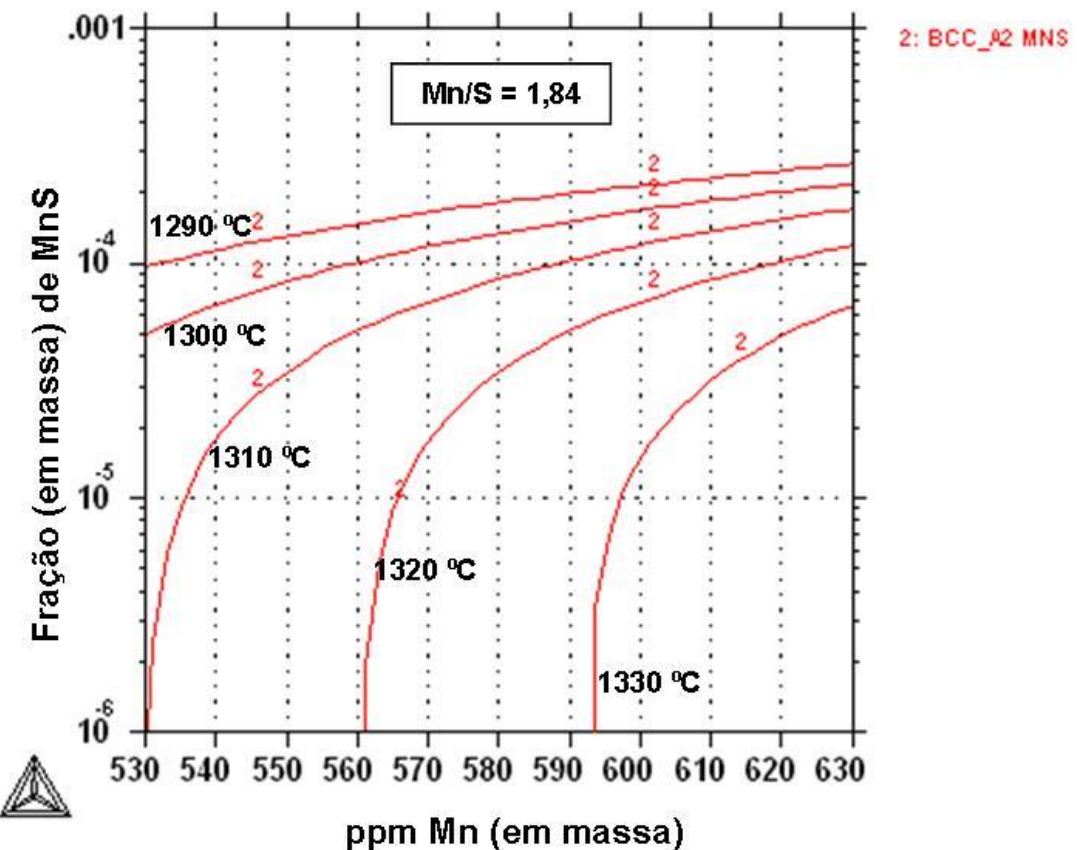

Figura 4. Fração (em massa) do inibidor Sulfeto de Manganês MnS, calculadas em várias temperaturas considerando razão $\mathrm{Mn} / \mathrm{S}=1,84$, em função do ppm de Manganês do aço elétrico $\mathrm{GO}$.

* Contribuição técnica ao $69^{\circ}$ Congresso Anual da ABM - Internacional e ao 14ํㅡㄹ ENEMET - Encontro Nacional de Estudantes de Engenharia Metalúrgica, de Materiais e de Minas, 21 a 25 de julho de 2014, São Paulo, SP, Brasil. 


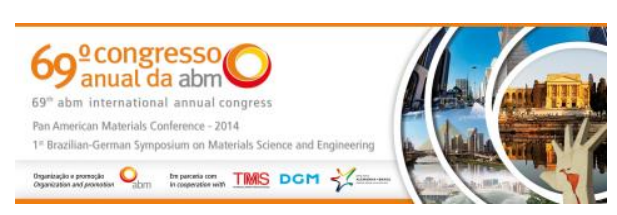

\subsection{Efeito da Atmosfera Gasosa na Oxidação Superficial dos Aços Elétricos}

Outra etapa fundamental do processo de fabricação do aço elétrico GO é a descarbonetação. Neste estágio, o aço é recozido em uma atmosfera controlada contendo os gases Hidrogênio $\left(\mathrm{H}_{2}\right)$ e Nitrogênio $\left(\mathrm{N}_{2}\right)$ como também $\mathrm{H}_{2} \mathrm{O}$ na fase líquida dissolvida nesses gases. O teor de Carbono no aço é reduzido a níveis inferiores a 20ppm [14] o que diminui muito a possibilidade de ocorrer precipitação de carbonetos que causam o fenômeno do envelhecimento magnético [15] em máquinas elétricas. Além disso, a superfície do aço em contato com a atmosfera oxida, formando uma subcamada de óxidos constituída por faialita e sílica. Posteriormente à etapa de descarbonetação o aço é revestido com uma camada de lama composta de $\mathrm{MgO}$ e aditivos. Esta lama além de atuar como separador refratário das espiras da bobina prevenindo a fusão das mesmas, ajuda a purificar o aço e ao reagir com a camada de óxidos estabelecida, forma a forsterita durante a etapa de recozimento final em caixa [16]. A função da forsterita é isolar eletricamente o aço, proteger contra a oxidações e reduzir a perda magnética devido a tensões aplicadas no aço, causadas pela a diferença de coeficiente de expansão térmica da forsterita e do aço elétrico [17].

Silveira et al. [18] investigam a formação desses óxidos sob diferentes condições de $\mathrm{pH}_{2} \mathrm{O} / \mathrm{pH}_{2}$ e temperatura de descarbonetação. Imagem de microscopia eletrônica de varredura de um micro corte na sessão transversal, Figura 5 mostram os aspectos morfológicos da subcamada de óxidos formada e os resultados das análises da espectroscopia por energia dispersiva de raio $X$ (EDX) realizadas em microscopia eletrônica de transmissão nas posições indicadas na Figura 6 sugerem as possíveis fases desses óxidos, Tabela 1.

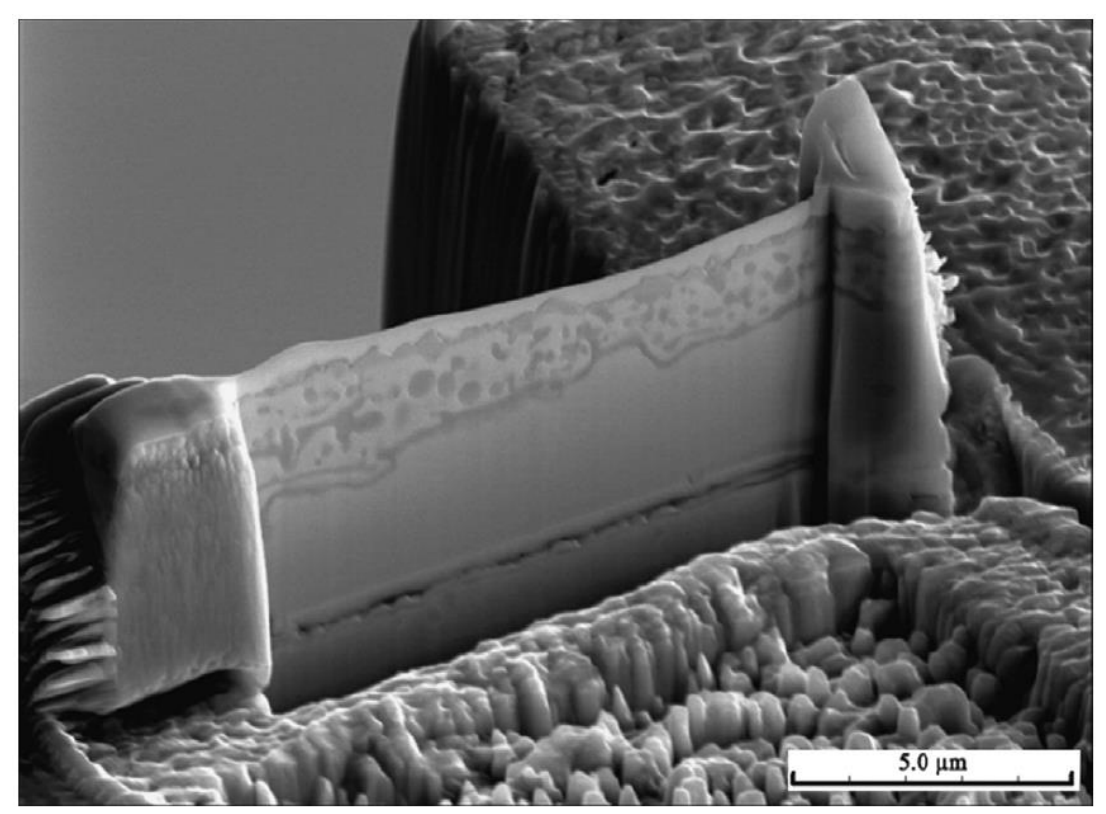

Figura 5. Imagem de microscopia eletrônica de varredura de um micro corte com 80nm de espessura na sessão transversal, realizado por um feixe de íons focalizados (FIB), mostrando os aspectos morfológicos da oxidação superficial do aço elétrico GO.

\footnotetext{
* Contribuição técnica ao $69^{\circ}$ Congresso Anual da ABM - Internacional e ao 14ํㅡㄹ ENEMET - Encontro Nacional de Estudantes de Engenharia Metalúrgica, de Materiais e de Minas, 21 a 25 de julho de 2014, São Paulo, SP, Brasil.
} 

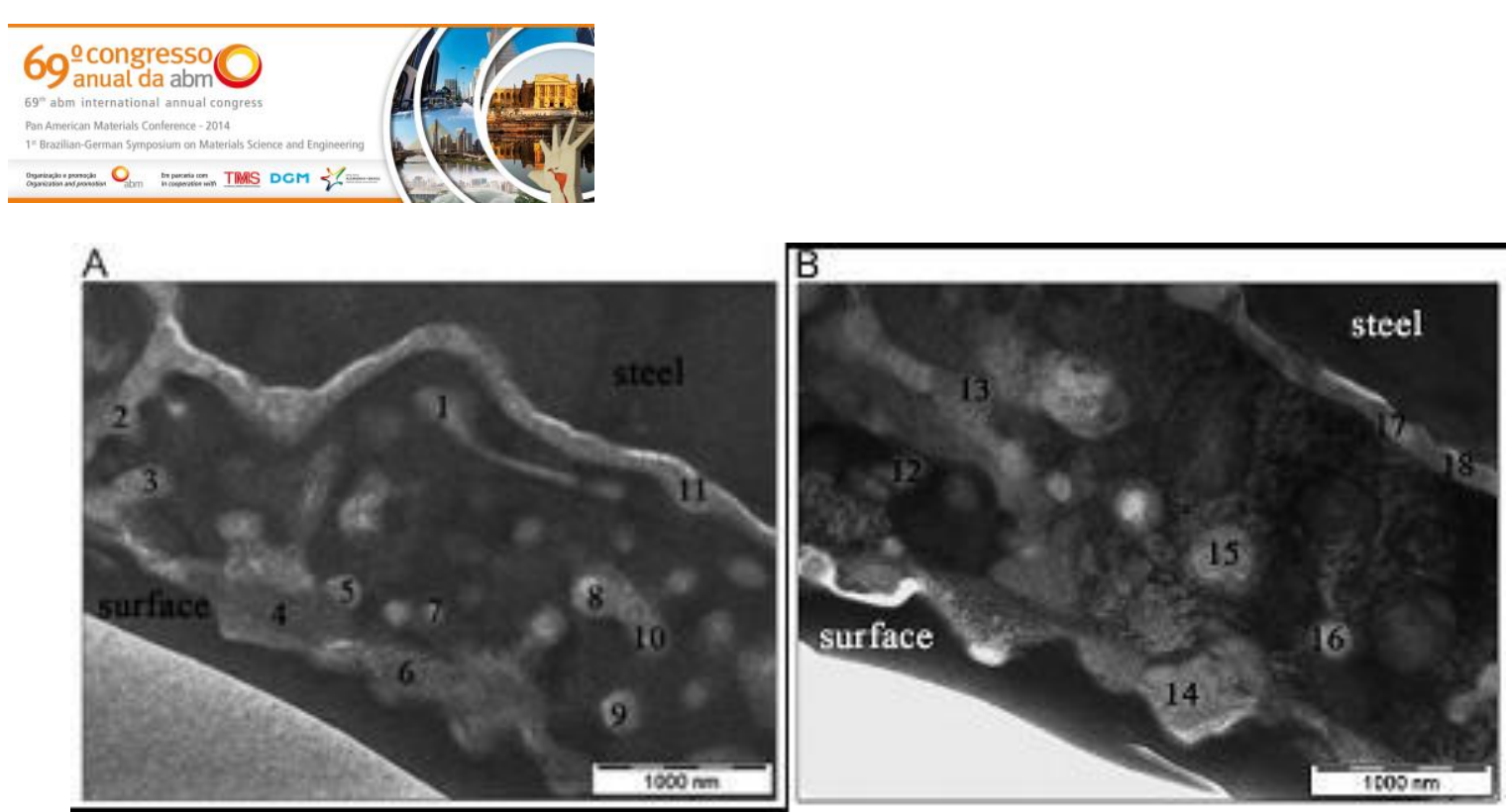

Figura 6. Imagem de microscopia eletrônica de transmissão da subcamada de óxido formada após a oxidação superficial do aço elétrico GO. Os números indicam a posição da sonda EDX cujos valores são mostrados na Tabela 1.

Tabela 1. Concentrações atômicas médias de Oxigênio, Silício e Ferro determinadas por espectroscopia de energia dispersiva de raio $X$ das posições indicadas na Figura 5

\begin{tabular}{|c|c|c|c|c|c|c|}
\hline Posição & \multicolumn{3}{|c|}{ At $\%$} & \multicolumn{2}{|c|}{ Razão Atômica } & Óxido \\
\hline & 0 & Si & $\mathrm{Fe}$ & $\mathrm{O} / \mathrm{Si}$ & $\mathrm{Fe} / \mathrm{Si}$ & \\
\hline $\begin{array}{l}1-3,5,7,8,12,13,15- \\
18\end{array}$ & 37 & 16 & 47 & 2,3 & 2,9 & $\mathrm{SiO} 2$ \\
\hline $9-11$ & 3 & 5 & 92 & 0,6 & 18,4 & $\mathrm{FeSiO} 4$ \\
\hline $4,6,14$ & 30 & 0 & 70 & - & - & $\mathrm{FeO}$ \\
\hline
\end{tabular}

O efeito da atmosfera descarbonetante foi calculado considerando uma atmosfera de $50 \% \mathrm{H}_{2}, 50 \% \mathrm{H}_{2}$ e $\mathrm{H}_{2} \mathrm{O}$ líquida dissolvida nesses gases. Para isso, para cada valor de temperatura investigada foi considerado sua correspondente atividade química do carbono, referente à fase grafita $(A C R(C, G R A))$, de uma liga de Fe-3,15\%Si-0,0375\%C. Desta forma para cada acréscimo de água dissolvida no gás, calculada através do ponto de orvalho, obtivemos o correspondente pressão parcial ou potencial químico do gás oxigênio $\left(\mathrm{pO}_{2}\right)$ da atmosfera para vários valores de temperatura, conforme mostrado na Figura 7.

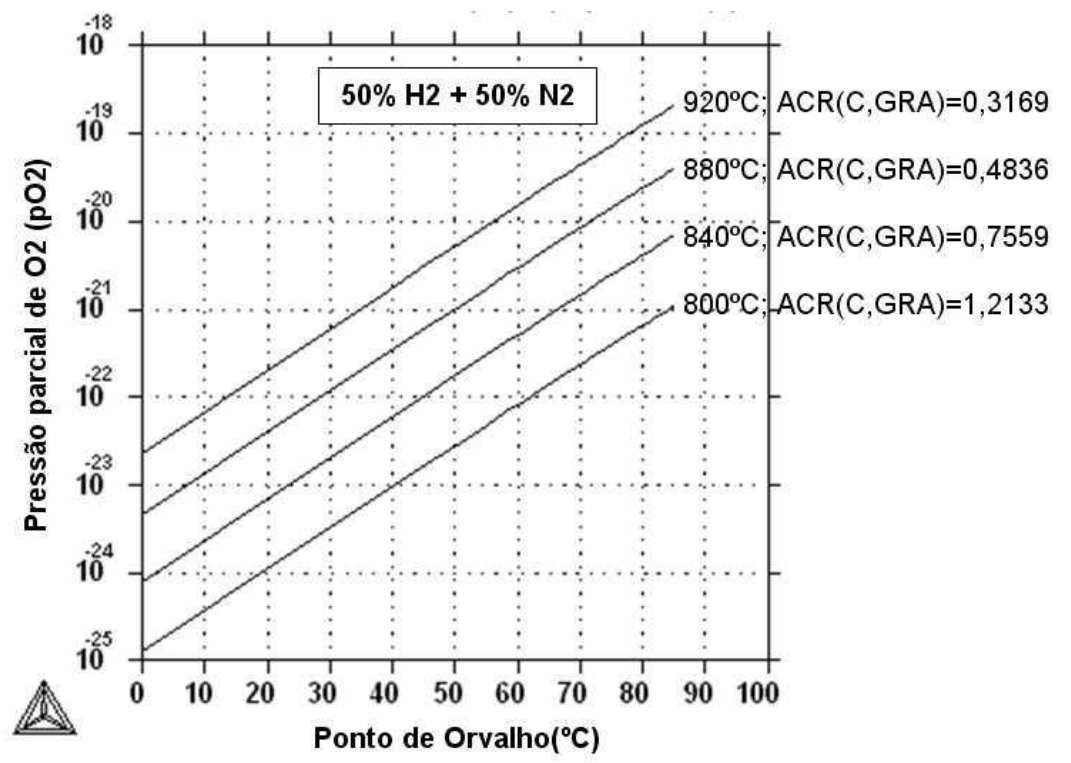

Figura 7. Pressão parcial do gás oxigênio $(\mathrm{pO} 2)$ para vários valores de ponto de orvalho de uma atmosfera com $50 \% \mathrm{H} 2$ e $50 \%$ N2 calculados para diferentes temperaturas.

\footnotetext{
* Contribuição técnica ao 69ำ Congresso Anual da ABM - Internacional e ao 14ํㅡㄹ ENEMET - Encontro Nacional de Estudantes de Engenharia Metalúrgica, de Materiais e de Minas, 21 a 25 de julho de 2014, São Paulo, SP, Brasil.
} 


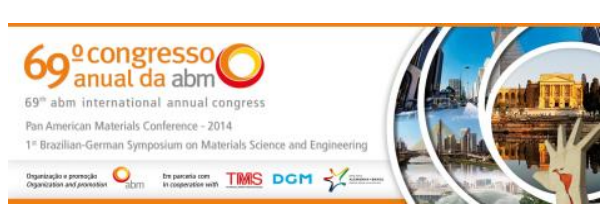

O teor (em ppm) de $\mathrm{O} 2$ dos óxidos formados quando a temperatura é $840^{\circ} \mathrm{C}$ em função da pressão parcial de $\mathrm{O} 2$ obtida na Figura 7 foi calculada os resultados são mostrados na Figura 8. Nota-se que os óxidos mais presentes nas condições mencionadas são $\mathrm{SiO} 2$ e a faialita.

Dependendo da pressão de ancoramento dos sulfetos, descrito na sessão 3.1, as condições da descarbonetação podem ser alteradas a fim de fomentar 0 desenvolvimento da textura Goss no aço elétrico GO. Embora as condições de descarbonetação sejam controladas, em termos de ponto de orvalho e temperatura. A formação de óxidos múltiplos verificadas nas Figuras 5 e 6 é atribuída a reações químicas onde o equilíbrio termodinâmico ainda não foi alcançado uma vez que as temperaturas da etapa de descarbonetação são moderadas e os tempos, em que o aço fica nesta temperatura sob ação da atmosfera são relativamente curtos (menores que 100 segundos).

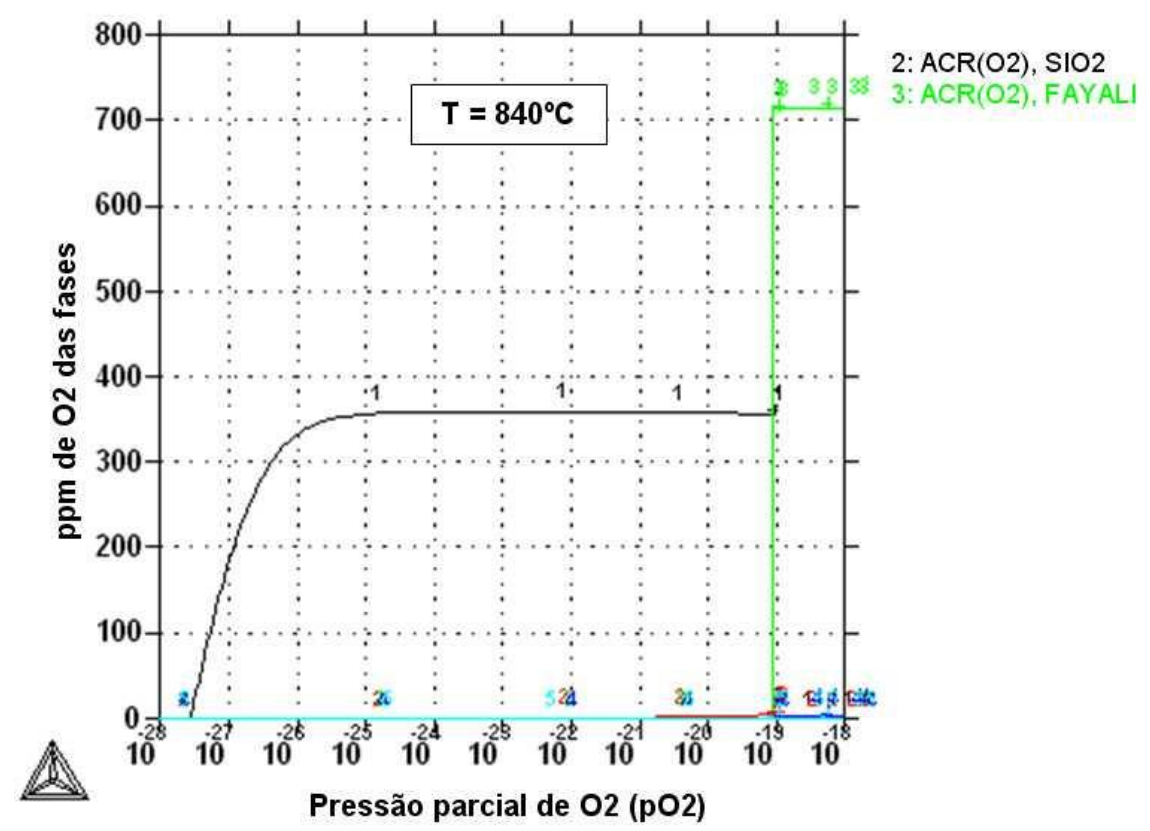

Figura 8. Teor de $\mathrm{O} 2$ (em ppm) em função da pressão parcial de $\mathrm{O} 2$ na temperatura de $840^{\circ} \mathrm{C}$

\section{CONCLUSÃO}

Os efeitos da composição química, da temperatura e da atmosfera gasosa nas transformações de fases que acontece durante as várias etapas de processamento dos aços elétricos foram avaliados. Diagramas de equilíbrio para as etapas de laminação a quente e descarbonetação foram calculados usando o software ThermoCalc versão 5.0 e os dados termodinâmicos dos sistemas estudados foram extraídos das bases de dados TCFE6 - TCS Steels/Fe-Alloys Database v6.2 e SSUB4 - SGTE Substance Database v4.1. Os resultados desses cálculos permitiram obter informações sobre as melhores alternativas para desenvolvimento desses aços uma vez que as faixas mínimas e máximas dos parâmetros do produto podem ser simuladas facilmente. As discrepâncias dos resultados calculados com os medidos experimentalmente podem ser atribuídas a fenômenos cinéticos ou que ocorreram fora do estado de equilíbrio termodinâmico.

Embora a obtenção de textura cristalográfica nos aços elétricos seja o parâmetro mais importante, e neste trabalho obtivemos informações de maneira indireta sem considerar o tempo, existem outros softwares capazes de realizar simulação cinética e microestrutural considerando diversas variáveis de entrada e condições de contorno. Estes softwares são: TC-Prisma e Micress.

* Contribuição técnica ao $69^{\circ}$ Congresso Anual da ABM - Internacional e ao 14ํㅡㄹ ENEMET - Encontro Nacional de Estudantes de Engenharia Metalúrgica, de Materiais e de Minas, 21 a 25 de julho de 2014, São Paulo, SP, Brasil. 


\section{Agradecimentos}

\section{LNAV agradece a Aperam South America pelo apoio.}

\section{REFERÊNCIAS}

1 The Economist. An inferno of unprofitability. The Economist Newspaper Limited; Jul 6, 2013.

2 National Science and Technology Council. Materials Genome Initiative for Global Competitiveness. Executive Office of the President of USA; Jun, 2011.

3 Costa e Silva A, Agren J, Clavaguera-Mora MT, Djurovic D, Gomez-Acebo T, Lee BJ, et al. Applications of computational thermodynamics - the extension from phase equilibrium to phase transformations and other properties. Calphad. 2007;31(1):53-74,

4 Aperam. Aperam Electrical Steels Catalog. 2013. Disponível em: http://www.aperam.com/brazil/port/arquivos/CatalogoAcosEletricosPORT.pdf

5 Viana CS, Lopes AM. Textura e relações de orientação. $2^{\underline{a}}$ ed. IPEN; 2003.

6 Ray RK, Jonas JJ. Transformation textures in steels Int. Mat. Rev. 1990;35(1):1-36.

7 Lima NEF, Lima FG, Toledo RA, Miranda LL, Paolinelli SC, Rodrigues MF, et al. Acesita technical report RT20-0112/2007. November 2007.

8 Landgraf FJG, Takanohashi R, Campos MF. Textura e relações de orientação. $2^{\mathrm{a}}$ ed. IPEN; 2003.

9 Hillert M. On the theory of normal and abnormal grain growth. Acta Metallurgica. 1965;13:227-238.

10 Cahn RW, Haasen P. Ptiysical Metallurgy. $3^{\underline{a}}$ ed. Nortti-Holland Ptiysics Publistiing;1983.

11 Nokuti Y, Maeda C. Trans. Iron Steel Inst., Jpn. 1984;24:657.

12 Sokolov BK. The interaction of grain boundaries and dispersed inclusions of the soluble phase. Fiz. Metal. Metalloved. 1977;43:1028-35.

13 Humphreys FJ, Hatherly M. Recrystallization and Related Annealing Phenomena. Australia: University of New South Wales; 2004.

14 Yamazaki T. On the decarburization of silicon steel sheet. Transactions ISIJ. 1969;9:6675.

15 Eloot K, Dilewinjins J, Standaert C, Cooman BC. Evaluation of the susceptibility of an electrical steel to magnetic aging using a new internal friction technique. Journal of Magnetism and Magnetic Materials. 1994;133:223-225.

16 Cesar MGMM, Vasconcelos CL, Vasconcelos WL. Microstructural characterization of magnesias derived from different sources and their influence on the structure of ceramic films formed on a 3\% silicon steel surface. Journal of Materials Science. 2002;37:232329.

17 Masui H, Shiozaki M, Takahashi N, Kabayashi H, Nagashima T, Yamazaki S, et al. Grain Oriented Silicon Steel Sheet Having Excellent Primary Film Properties. Nippon Steel Corporation, Japan. Int. Cl6. B32B15/04. U. S. P. n 5,565,272. 10 Jul. 1992; 15 Oct. 1996.

18 Silveira CC, Cunha MA, Buono VTL. The influence of internal oxidation during decarburization of a grain oriented silicon steel on the morphology of the glass film formed at high temperature annealing. Journal of Magnetism and Magnetic Materials. 2014;358-359:65-69.

\footnotetext{
* Contribuição técnica ao $69^{\circ}$ Congresso Anual da ABM - Internacional e ao 14ํㅡㄹ ENEMET - Encontro Nacional de Estudantes de Engenharia Metalúrgica, de Materiais e de Minas, 21 a 25 de julho de 2014, São Paulo, SP, Brasil.
} 